\title{
The stability and bifurcation analysis of a discrete Holling-Tanner model
}

\author{
Hui Cao ${ }^{{ }^{*}}$, Zongmin Yue ${ }^{1}$ and Yicang Zhou ${ }^{2}$
}

"Correspondence: caohui@sust.edu.cn

'Department of Mathematics, Shaanxi University of Science \& Technology, Xi'an, 710021, China Full list of author information is available at the end of the article

\begin{abstract}
A discrete predator-prey model with Holling-Tanner functional response is formulated and studied. The existence of the positive equilibrium and its stability are investigated. More attention is paid to the existence of a flip bifurcation and a Neimark-Sacker bifurcation. Sufficient conditions for those bifurcations have been obtained. Numerical simulations are conducted to demonstrate our theoretical results and the complexity of the model.
\end{abstract}

Keywords: discrete Holling-Tanner model; flip bifurcation; Neimark-Sacker bifurcation; stability

\section{Introduction}

Differential equations and difference equations are two typical mathematical approaches to modeling population dynamical systems. There have been an increasing interest and research results on discrete population dynamical systems in spite of their complexity [1-4].

Predator-prey models describe one of the most important relationships between two interacting species and have received much attention of applied mathematicians and ecologists. The stability and existence of equilibrium state, the permanence of a system, the Hopf bifurcation and the chaos of different continuous predator-prey models have been extensively investigated. However, there are less results on dynamical behaviors of discrete predator-prey models. The flip bifurcation and the Neimark-Sacker bifurcation are two important phenomena of discrete population model dynamics. Liu and Xiao [5] used the center manifold theorem to study the flip bifurcation and the Neimark-Sacker bifurcation. Agiza et al. [2] and Celik et al. [6] used the numerical simulations to discuss the flip bifurcation and the Neimark-Sacker bifurcation. Hu et al. [4] also used the center manifold theorem to study the flip bifurcation and the Neimark-Sacker bifurcation.

The following continuous prey-predator model with Holling-Tanner functional response is very interesting and has been studied by many authors [7-11]:

$$
\begin{aligned}
& \frac{d x}{d t}=r_{1} x\left(1-\frac{x}{K}\right)-\frac{q x y}{x+a}, \\
& \frac{d y}{d t}=r_{2} y\left(1-\frac{y}{\gamma x}\right),
\end{aligned}
$$

@2013 Cao et al.; licensee Springer. This is an Open Access article distributed under the terms of the Creative Commons Attribution License (http://creativecommons.org/licenses/by/2.0), which permits unrestricted use, distribution, and reproduction in any medium, provided the original work is properly cited. 
where $x(t)$ and $y(t)$ are the numbers of the prey and the predator species at time $t$, respectively. $r_{1}$ and $r_{2}$ are the intrinsic growth rates or biotic potential of the prey and predator, respectively. $K$ is the prey environment carrying capacity. $\gamma$ is a measure of the food quality that the prey provides for conversion into predator births. $q$ is the maximal predator per capita consumption. $a$ is the number of prey necessary to achieve one-half of the maximum rate $q$. The variables and parameters satisfy $(x, y) \in\{(x, y) \mid x>0, y>0\}$ and $r_{1}, r_{2}, K, \gamma, q, a>0$.

After introducing the new variables and parameters

$$
u=\frac{x}{K}, \quad v=\frac{y}{\gamma K}, \quad \tau=r_{1} t, \quad \theta=\frac{r_{1}}{r_{2}}, \quad b=\frac{a}{K}, \quad c=\frac{q \gamma}{r_{1}},
$$

system (1) becomes

$$
\begin{aligned}
& \frac{d u}{d \tau}=u(1-u)-\frac{c u v}{u+b}, \\
& \frac{d v}{d \tau}=\theta v\left(1-\frac{v}{u}\right)
\end{aligned}
$$

Motivated by a similar idea, we study the following discrete-time model corresponding to model (2):

$$
\begin{aligned}
& u(t+1)=u(t) \exp \left[1-u(t)-\frac{c v(t)}{u(t)+b}\right] \\
& v(t+1)=v(t) \exp \left[\theta\left(1-\frac{v(t)}{u(t)}\right)\right],
\end{aligned}
$$

where $u, v, c, b$, and $\theta$ are defined as in model (2). It is assumed that the initial value of solutions of system (3) satisfies $u(0)>0, v(0)>0$ and all the parameters are positive. It is easy to prove that if the initial values $(u(0), v(0))$ are positive, then the corresponding solution $(u(t), v(t))$ is positive too.

In this paper, we study the dynamical behaviors of model (3). The existence and stability of the positive equilibrium are investigated in Section 2. The criteria for the existence of a flip bifurcation and a Neimark-Sacker bifurcation are given in Section 3. Numerical simulations are conducted to demonstrate our theoretical results and show the complexity of the model dynamics in Section 3, too. Concluding remarks and discussions are given in Section 4.

\section{The existence and stability of the equilibrium}

We firstly discuss the existence of the equilibria of model (3). From model (3) we know that the coordinates $u$ and $v$ of the positive equilibrium satisfy

$$
1-u-\frac{c v}{u+b}=0, \quad 1-\frac{v}{u}=0,
$$

which is equivalent to

$$
v=u, \quad u^{2}+(b+c-1) u-b=0 .
$$


Quadratic equation (5) has a positive solution

$$
u_{*}=\frac{-(b+c-1)+\sqrt{(b+c-1)^{2}+4 b}}{2} .
$$

Then $E\left(u_{*}, u_{*}\right)$ is a positive equilibrium of model (3). From the expression

$$
u_{*}=\frac{-(b+c-1)+\sqrt{(b+c-1)^{2}+4 b}}{2}=\frac{2 b}{b+c-1+\sqrt{(b+c-1)^{2}+4 b}},
$$

we know that $u_{*}$ is a decreasing function of $c$ with $\lim _{c \rightarrow 0^{+}} u_{*}=1, \lim _{c \rightarrow \infty} u_{*}=0$, and $0<u_{*}<1$.

The linearized matrix of model (3) at the equilibrium $E\left(u_{*}, u_{*}\right)$ is

$$
J=\left(\begin{array}{cc}
1-u_{*}+\frac{c u_{*}^{2}}{\left(u_{*}+b\right)^{2}} & -\frac{c u_{*}}{u_{*}+b} \\
\theta & 1-\theta
\end{array}\right) .
$$

The characteristic equation of matrix $J$ is $h(\lambda)=0$, with $h(\lambda)=\lambda^{2}-p \lambda+q=0$, where

$$
\begin{aligned}
& p=2-\theta-u_{*}+\frac{c u_{*}^{2}}{\left(u_{*}+b\right)^{2}}, \\
& q=1-\theta-u_{*}+\theta u_{*}+\frac{c u_{*}\left(u_{*}+b \theta\right)}{\left(u_{*}+b\right)^{2}} .
\end{aligned}
$$

Let $\lambda_{1}$ and $\lambda_{2}$ be two solutions of $h(\lambda)=0$, and $\Lambda=\max \left\{\left|\lambda_{1}\right|,\left|\lambda_{2}\right|\right\}$. From the Jury criterion, we know that the necessary and sufficient conditions for $\Lambda<1$ are

$$
1+p-q>0, \quad 1-p+q>0, \quad 1-q>0 .
$$

It is easy to obtain that

$$
1-p+q=\theta u_{*}+\frac{b c \theta u}{\left(u_{*}+b\right)^{2}}>0
$$

holds true for all positive parameters. The other two conditions become

$$
\begin{aligned}
& 1+p+q=4-2 \theta-2 u_{*}+\theta u_{*}+\frac{2 c u_{*}^{2}}{\left(u_{*}+b\right)^{2}}+\frac{b c \theta u_{*}}{\left(u_{*}+b\right)^{2}}>0, \\
& 1-q=u_{*}+\theta-u \theta-\frac{c u_{*}^{2}}{\left(u_{*}+b\right)^{2}}-\frac{b c \theta u_{*}}{\left(u_{*}+b\right)^{2}}>0 .
\end{aligned}
$$

The conditions $1+p+q>0$ and $1-q>0$ in (6) are equivalent to

$$
\frac{\left(2 \theta+2 u_{*}-4-\theta u_{*}\right)\left(u_{*}+b\right)^{2}}{2 u_{*}^{2}+b \theta u_{*}}<c<\frac{\left(\theta+u_{*}-\theta u_{*}\right)\left(u_{*}+b\right)^{2}}{u_{*}^{2}+b \theta u_{*}} .
$$

By using the equation $\frac{c u_{*}}{u_{*}+b}=1-u_{*}$ and $u_{*}^{2}=b-(b+c-1) u_{*}$, we can have another equivalent conditions of (6)

$$
\frac{1-b-2 u_{*}}{1-u_{*}}<\theta<2+\frac{2 c}{b+c+1} .
$$


Then we have the following stability theorem.

Theorem 2.1 The unique positive equilibrium point $E\left(u_{*}, u_{*}\right)$ of model (3) is asymptotically stable if and only if condition (7) or condition (8) holds.

Proof From the straightforward calculation, we can have the equivalent condition given in (7). Here we verify the equivalence of condition (6) and condition (8). The first inequality in condition (6) is equivalent to

$$
\frac{c \theta u_{*}}{u_{*}+b}>(\theta-2)\left(2-u_{*}+\frac{c u_{*}^{2}}{\left(u_{*}+b\right)^{2}}\right) \text {. }
$$

Substituting $\frac{c u_{*}}{u_{*}+b}=1-u_{*}$ into inequality (9) yields

$$
\theta\left(1-u_{*}\right)>(\theta-2)\left(2-u_{*}+\frac{u_{*}\left(1-u_{*}\right)}{u_{*}+b}\right) .
$$

Inequality in (10) is equivalent to

$$
2\left(u_{*}+b+\left(1-u_{*}\right)\left(2 u_{*}+b\right)\right)>\theta\left(u_{*}+b+\left(1-u_{*}\right) u_{*}\right) .
$$

Using the equality $u_{*}^{2}=b-(b+c-1) u_{*}$ in (11) leads to

$$
2 u_{*}(1+b+2 c)>\theta(1+b+c) u_{*} .
$$

It follows from (12) that

$$
\theta<\frac{2(1+b+2 c)}{1+b+c)}=2+\frac{2 c}{1+b+c} .
$$

The second inequality in (6) is equivalent to

$$
(1-\theta)\left(1-u_{*}+\frac{c u_{*}^{2}}{\left(u_{*}+b\right)^{2}}\right)<1-\frac{c \theta u_{*}}{u_{*}+b} .
$$

Substituting $\frac{c u_{*}}{u_{*}+b}=1-u_{*}$ into inequality (14) yields

$$
(1-\theta)\left(1-u_{*}+\frac{u_{*}\left(1-u_{*}\right)}{u_{*}+b}\right)<1-\theta\left(1-u_{*}\right) .
$$

Inequality in (15) is equivalent to

$$
u_{*}\left(1-u_{*}\right)-u_{*}\left(u_{*}+b\right)<\theta\left(1-u_{*}\right) u_{*} .
$$

From inequality (16) and $0<u_{*}<1$ it follows that

$$
\theta>\frac{1-b-2 u_{*}}{1-u_{*}} .
$$

We can have inequality in condition (8) by combining inequalities (13) and (17). 

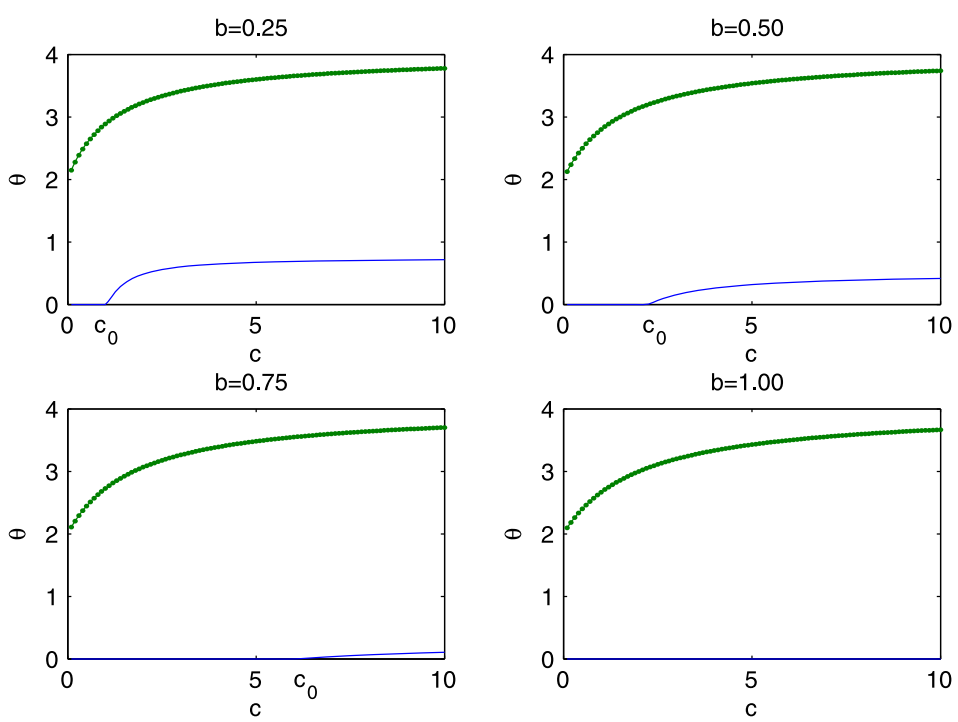

Figure 1 The stability domain of the equilibrium $E\left(u_{*}, u_{*}\right)$ of model (3).

Remark 1 Inequality (7) or (8) gives stability conditions for the equilibrium $E\left(u_{*}, u_{*}\right)$ of model (3). Inequality (7) is directly obtained from (6), but it is not easy to verify since $u_{*}$ is dependent on $c$. As stated in the proof of Theorem 2.1, inequality (8) is easy to verify though it is difficult to obtain.

Remark 2 When $1-b-2 u_{*}<0$, equivalent to $2(1-b) c<(1+b)^{2}$, the inequality $\theta>\frac{1-b-2 u_{*}}{1-u_{*}}$ holds true automatically. The stability condition becomes $\theta<2+\frac{2 c}{1+b+c}$, which is easy to verify.

For $b=\frac{1}{4}, b=\frac{1}{2}, b=\frac{3}{4}$, and $b=1$, the stability domain in the $c-\theta$ plane is shown in Figure 1 . The horizontal and vertical coordinates are the parameters $c$ and $\theta$, respectively. For any given $c$, the positive equilibrium $E\left(u_{*}, u_{*}\right)$ is stable when $\theta$ is between two given curves. There exists $c_{0}$ for the subplots with $b=\frac{1}{4}, b=\frac{1}{2}$, and $b=\frac{3}{4}$, respectively. When $c<c_{0}$, the positive equilibrium $E\left(u_{*}, u_{*}\right)$ of model (3) is stable for $0<\theta<2+\frac{2 c}{1+b+c}$. When $c>c_{0}$, the positive equilibrium $E\left(u_{*}, u_{*}\right)$ of model (3) is stable for $\frac{1-b-2 u_{*}}{1-u_{*}}<\theta<2+\frac{2 c}{b+c+1}$.

From conditions given in Theorem 2.1 we know that the positive equilibrium $E\left(u_{*}, u_{*}\right)$ of model (3) is locally stable if $\frac{1-b-2 u_{*}}{1-u_{*}}<\theta<2+\frac{2 c}{b+c+1}$. The numerical simulations demonstrate that the positive equilibrium $E\left(u_{*}, u_{*}\right)$ of model (3) may be globally asymptotically stable if the conditions in Theorem 2.1 hold. If we take $b=0.5$ and $c=5$, then $E(0.1085,0.1085)$ is the positive equilibrium of model (3). The stability condition becomes $0.3175<\theta<3.5384$. If we take $\theta=0.6$, then $\lambda_{1}=\bar{\lambda}_{2}=0.7252+0.6551 i$ are the complex eigenvalues of the linearized matrix $J$ of model (3) at the positive equilibrium $E(0.1085,0.1085) . E(0.1085,0.1085)$ is a stable focus. For the initial value $u(0)=0.1$ and $v(0)=1.2$, the solution series of $u(t)$ and the phase portrait are given in Figure 2 (the left column of subplots). If we take $\theta=0.3 .48$, then $\lambda_{1}=-0.5981$ and $\lambda_{2}=-0.8314$ are the real eigenvalues of the linearized matrix $J$ of model (3) at the positive equilibrium $E(0.1085,0.1085) . E(0.1085,0.1085)$ is a stable node. For the same initial value $u(0)=0.1$ and $v(0)=1.2$, the solution series of $u(t)$ and $v(t)$ are given in Figure 2 (the right column 

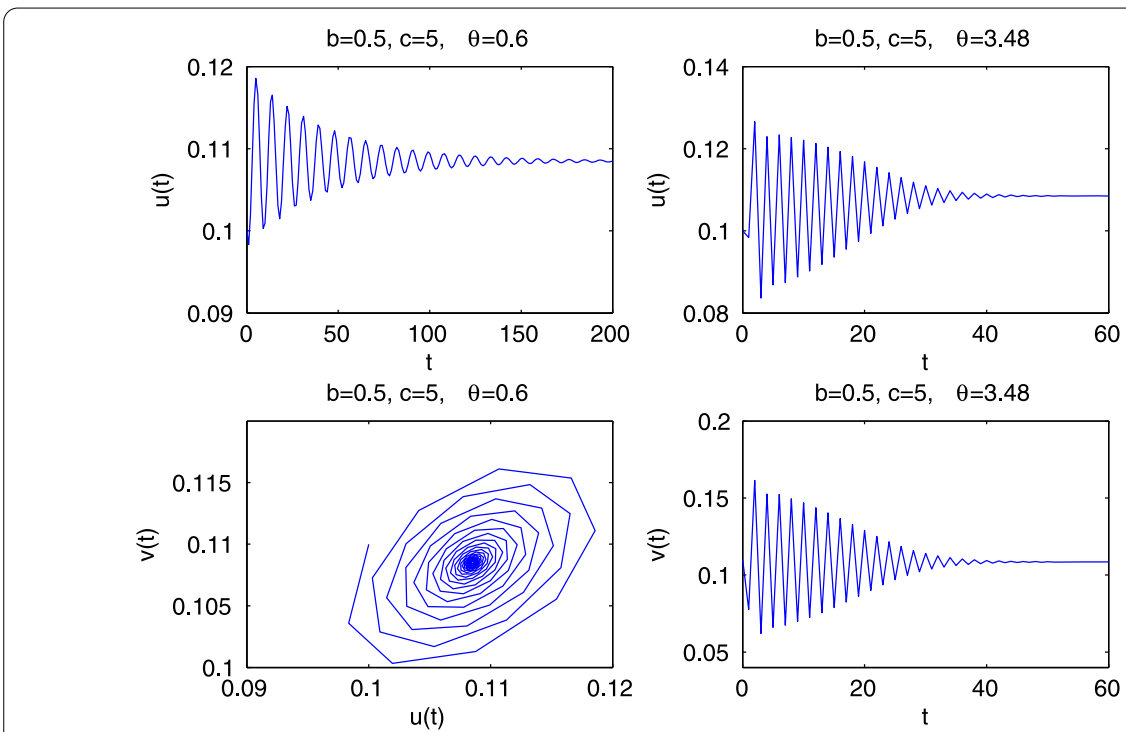

Figure 2 The stability of the equilibrium $E\left(u_{*}, u_{*}\right)$ of model (3).

of subplots). The solution series and the phase portrait in Figure 2 show that the positive equilibrium $E(0.1085,0.1085)$ of model (3) may be globally asymptotically stable.

\section{Bifurcation}

Bifurcation may lead to different dynamical behaviors of a model when parameters pass through a critical values. Bifurcation usually occurs when the stability of an equilibrium changes. In this section, we discuss the flip bifurcation and the Neimark-Sacker bifurcation of model (3).

\subsection{Flip bifurcation}

We define $\theta_{*}=2+\frac{2 c}{b+c+1}$. The stability analysis in Section 3 shows that the positive equilibrium $E\left(u_{*}, u_{*}\right)$ has an eigenvalue -1 when $\theta=\theta_{*}$, which means $E\left(u_{*}, u_{*}\right)$ is non-hyperbolic. The flip bifurcation may occur in the neighborhood of the endemic equilibrium $E\left(u_{*}, u_{*}\right)$ when $\theta$ passes through the critical point $\theta_{*}$.

The linearization matrix of model (3) at the equilibrium point $E\left(u_{*}, u_{*}\right)$ with $\theta=\theta_{*}$ is

$$
A=\left(\begin{array}{cc}
1-u_{*}+\frac{c u_{*}^{2}}{\left(u_{*}+b\right)^{2}} & -\frac{c u_{*}}{u_{*}+b} \\
2+\frac{2 c}{b+c+1} & -1-\frac{2 c}{b+c+1}
\end{array}\right),
$$

and the characteristic equation of matrix $A$ is $\lambda^{2}+p_{*} \lambda+q_{*}=0$, where

$$
\begin{aligned}
& p_{*}=\frac{\left(2 u_{*}+b-1\right) u_{*}}{u_{*}+b}+\frac{2 c}{b+c+1}, \\
& q_{*}=\left(1-u_{*}\right)\left(1-\frac{u_{*}}{u_{*}+b}\left(1+\frac{2 c}{b+c+1}\right)\right) .
\end{aligned}
$$

The eigenvalues of matrix $A$ are $\lambda_{1}=-1$ and $\lambda_{2}=\frac{(b+2 c)(b+c)+b}{1+b+c}-\frac{b(1+b+2 c)}{u_{*}+b}$ with $\left|\lambda_{2}\right| \neq 1$. The following theorem confirms the flip bifurcation of model (3). 
Theorem 3.1 If $\beta \neq 0$, then model (3) will undergo a flip bifurcation at $E\left(u_{*}, u_{*}\right)$ when $\theta=\theta_{*}$. That is, there exists a stable period two cycle if $\theta_{*}<\theta<\theta_{*}+\varepsilon$, where $\varepsilon$ is a small positive number, and $\beta$ is defined in the end of the proof.

Proof In order to use the center manifold theory, we treat $\theta$ as a state variable. The transformations $\tilde{u}=u-u_{*}, \tilde{v}=v-u_{*}$, and $\tilde{\theta}=\theta-\theta_{*}$ take model (3) into the form

$$
\begin{aligned}
& \tilde{u}(t+1)=\left(\tilde{u}(t)+u_{*}\right) \exp \left(1-\tilde{u}(t)-u_{*}-\frac{c\left(\tilde{v}(t)+u_{*}\right)}{\tilde{u}(t)+u_{*}+b}\right)-u_{*}, \\
& \tilde{\theta}(t+1)=\tilde{\theta}(t), \\
& \tilde{v}(t+1)=\left(\tilde{v}(t)+u_{*}\right) \exp \left(\left(\tilde{\theta}(t)+\theta_{*}\right)\left(1-\frac{\tilde{v}(t)+u_{*}}{\tilde{u}(t)+u_{*}}\right)\right)-u_{*} .
\end{aligned}
$$

Taylor expansion of model $(18)$ at $(\tilde{u}, \tilde{v}, \tilde{\theta})=(0,0,0)$ is

$$
\begin{aligned}
& \tilde{u}(t+1)=\frac{(b+2 c) u_{*}-b}{u_{*}+b} \tilde{u}(t)-\left(1-u_{*}\right) \tilde{v}(t)+F_{1}(\tilde{u}, \tilde{v}, \tilde{\theta}), \\
& \tilde{\theta}(t+1)=\tilde{\theta}(t) \\
& \tilde{v}(t+1)=\theta_{*} \tilde{u}(t)+\left(1-\theta_{*}\right) \tilde{v}(t)+F_{2}(\tilde{u}, \tilde{v}, \tilde{\theta}),
\end{aligned}
$$

where

$$
\begin{aligned}
F_{1}(\tilde{u}, \tilde{v}, \tilde{\theta}) & \\
= & \frac{\left(2 c-1-(b+2 c)\left(b+2 u_{*}\right)\right) u_{*}}{2\left(u_{*}+b\right)^{2}} \tilde{u}^{2}+\frac{(b+2 c) u_{*}-(b+c)}{u_{*}+b} \tilde{u} \tilde{v}+\frac{c\left(1-u_{*}\right)}{2\left(u_{*}+b\right)} \tilde{v}^{2} \\
& +\frac{6\left(1-u_{*}\right)\left[b+(1-b-2 c) u_{*}\right]+\left(1-b-2 u_{*}\right)^{2}\left[b+(2+b+2 c) u_{*}\right]}{6\left(u_{*}+b\right)^{3}} \tilde{u}^{3} \\
& +\frac{2\left[c-u_{*}\left(1-u_{*}\right)\right]+\left(1-b-2 u_{*}\right)\left[\left(1-u_{*}\right)\left(1+b+2 u_{*}\right)-2 c\right]}{2\left(u_{*}+b\right)^{2}} \tilde{u}^{2} \tilde{v} \\
& +\frac{c\left[c-(1-u)\left(1+b+2 u_{*}\right)\right]}{2\left(u_{*}+b\right)^{2}} \tilde{u}^{2}-\frac{c^{2}\left(1-u_{*}\right)}{6\left(u_{*}+b\right)^{2}} \tilde{v}^{3}+o\left((|\tilde{u}|+|\tilde{v}|+|\tilde{\theta}|)^{3}\right),
\end{aligned}
$$

$F_{2}(\tilde{u}, \tilde{v}, \tilde{\theta})$

$$
\begin{aligned}
= & \frac{\theta_{*}\left(\theta_{*}-2\right)}{2 u_{*}} \tilde{u}^{2}+\frac{2 \theta_{*}-\theta_{*}^{2}}{u_{*}} \tilde{u} \tilde{v}+\tilde{u} \tilde{\theta}+\frac{\theta_{*}^{2}-2 \theta_{*}}{2 u_{*}} \tilde{v}^{2}-\tilde{v} \tilde{\theta}+\frac{6 \theta_{*}-6 \theta_{*}^{2}+\theta_{*}^{3}}{6 u_{*}^{2}} \tilde{u}^{3} \\
& +\frac{-4 \theta_{*}+5 \theta_{*}^{2}-\theta_{*}^{3}}{2 u_{*}^{2}} \tilde{u}^{2} \tilde{v}+\frac{\theta_{*}-1}{u_{*}} \tilde{u}^{2} \tilde{\theta}+\frac{2 \theta_{*}-4 \theta_{*}^{2}+\theta_{*}^{3}}{2 u_{*}^{2}} \tilde{u} \tilde{v}^{2} \\
& +\frac{2-2 \theta_{*}}{u_{*}} \tilde{u} \tilde{v} \tilde{\theta}+\frac{\theta_{*}-1}{u_{*}} \tilde{v}^{2} \tilde{\theta}+\frac{3 \theta_{*}^{2}-\theta_{*}^{3}}{6 u_{*}^{2}} \tilde{v}^{3}+o\left((|\tilde{u}|+|\tilde{v}|+|\tilde{\theta}|)^{3}\right) .
\end{aligned}
$$

We define the matrix

$$
T=\left(\begin{array}{ccc}
1 & 0 & 1 \\
0 & 1 & 0 \\
\frac{\theta_{*}}{\theta_{*}-2} & 0 & \frac{\theta_{*}}{\lambda_{2}-1+\theta_{*}}
\end{array}\right) \quad \text { and } \quad T^{-1}=\left(\begin{array}{ccc}
-\frac{\theta_{*}-2}{1+\lambda_{2}} & 0 & \frac{\left(\lambda_{2}-1+\theta_{*}\right)\left(\theta_{*}-2\right)}{\theta_{*}\left(1+\lambda_{2}\right)} \\
0 & 1 & 0 \\
\frac{\lambda_{2}-1+\theta_{*}}{1+\lambda_{2}} & 0 & -\frac{\left(\lambda_{2}-1+\theta_{*}\right)\left(\theta_{*}-2\right)}{\theta_{*}\left(1+\lambda_{2}\right)}
\end{array}\right)
$$


The transformation $\left(\begin{array}{c}\tilde{u}(t) \\ \tilde{\theta}(t) \\ \tilde{\nu}(t)\end{array}\right)=T\left(\begin{array}{c}u_{1}(t) \\ \theta_{1}(t) \\ v_{1}(t)\end{array}\right)$ takes model (19) to

$$
\left(\begin{array}{c}
u_{1}(t+1) \\
\theta_{1}(t+1) \\
v_{1}(t+1)
\end{array}\right)=\left(\begin{array}{ccc}
-1 & 0 & 0 \\
0 & 1 & 0 \\
0 & 0 & \lambda_{2}
\end{array}\right)\left(\begin{array}{c}
u_{1}(t) \\
\theta_{1}(t) \\
v_{1}(t)
\end{array}\right)+\left(\begin{array}{c}
Q_{1}\left(u_{1}, \theta_{1}, v_{1}\right) \\
0 \\
Q_{2}\left(u_{1}, \theta_{1}, v_{1}\right)
\end{array}\right)
$$

where

$$
\begin{aligned}
& Q_{1}\left(u_{1}, v_{1}, \theta_{1}\right)=-\frac{\theta_{*}-2}{1+\lambda_{2}} H_{1}\left(u_{1}, v_{1}, \theta_{1}\right)+\frac{\left(\lambda_{2}-1+\theta_{*}\right)\left(\theta_{*}-2\right)}{\theta_{*}\left(1+\lambda_{2}\right)} H_{2}\left(u_{1}, v_{1}, \theta_{1}\right), \\
& Q_{2}\left(u_{1}, v_{1}, \theta_{1}\right)=\frac{\lambda_{2}-1+\theta_{*}}{1+\lambda_{2}} H_{1}\left(u_{1}, v_{1}, \theta_{1}\right)-\frac{\left(\lambda_{2}-1+\theta_{*}\right)\left(\theta_{*}-2\right)}{\theta_{*}\left(1+\lambda_{2}\right)} H_{2}\left(u_{1}, v_{1}, \theta_{1}\right),
\end{aligned}
$$

with

$$
\begin{aligned}
H_{1}\left(u_{1}, v_{1}, \theta_{1}\right)= & a_{200} u_{1}^{2}+a_{110} u_{1} v_{1}+a_{020} v_{1}^{2}+a_{300} u_{1}^{3}+a_{210} u_{1}^{2} v_{1}+a_{120} u_{1} v_{1}^{2}+a_{030} v_{1}^{3} \\
& +o\left(\left(\left|u_{1}\right|+\left|v_{1}\right|+\left|\theta_{1}\right|\right)^{3}\right), \\
H_{2}\left(u_{1}, v_{1}, \theta_{1}\right)= & b_{200} u_{1}^{2}+b_{110} u_{1} v_{1}+b_{101} u_{1} \theta_{1}+b_{020} v_{1}^{2}+b_{011} v_{1} \theta_{1}+b_{300} u_{1}^{3}+b_{210} u_{1}^{2} v_{1} \\
& +b_{201} u_{1}^{2} \theta_{1}+b_{120} u_{1} v_{1}^{2}+b_{111} u_{1} v_{1} \theta_{1}+b_{030} v_{1}^{3}+b_{021} v_{1}^{2} \theta_{1} \\
& +o\left(\left(\left|u_{1}\right|+\left|v_{1}\right|+\left|\theta_{1}\right|\right)^{3}\right),
\end{aligned}
$$

and

$$
\begin{aligned}
& a_{200}=\frac{\left(2 c-1-(b+2 c)\left(b+2 u_{*}\right)\right) u_{*}}{2\left(u_{*}+b\right)^{2}}+\frac{\left((b+2 c) u_{*}-(b+c)\right) \theta_{*}}{\left(u_{*}+b\right)\left(\theta_{*}-2\right)}+\frac{c\left(1-u_{*}\right) \theta_{*}^{2}}{2\left(u_{*}+b\right)\left(\theta_{*}-2\right)^{2}}, \\
& a_{110}=\frac{\left(2 c-1-(b+2 c)\left(b+2 u_{*}\right)\right) u_{*}}{\left(u_{*}+b\right)^{2}}+\frac{\left((b+2 c) u_{*}-(b+c)\right) \theta_{*}}{\left(u_{*}+b\right)\left(\lambda_{2}-1+\theta_{*}\right)} \\
& +\frac{\left((b+2 c) u_{*}-(b+c)\right) \theta_{*}}{\left(u_{*}+b\right)\left(\theta_{*}-2\right)}+\frac{c\left(1-u_{*}\right) \theta_{*}^{2}}{\left(u_{*}+b\right)\left(\theta_{*}-2\right)\left(\lambda_{2}-1+\theta_{*}\right)}, \\
& a_{020}=\frac{\left(2 c-1-(b+2 c)\left(b+2 u_{*}\right)\right) u_{*}}{2\left(u_{*}+b\right)^{2}}+\frac{\left((b+2 c) u_{*}-(b+c)\right) \theta_{*}}{\left(u_{*}+b\right)\left(\lambda_{2}-1+\theta_{*}\right)} \\
& +\frac{c\left(1-u_{*}\right) \theta_{*}^{2}}{2\left(u_{*}+b\right)\left(\lambda_{2}-1+\theta_{*}\right)^{2}} \\
& a_{300}=\frac{6\left(1-u_{*}\right)\left[b+(1-b-2 c) u_{*}\right]+\left(1-b-2 u_{*}\right)^{2}\left[b+(2+b+2 c) u_{*}\right]}{6\left(u_{*}+b\right)^{3}} \\
& +\frac{c\left[c-(1-u)\left(1+b+2 u_{*}\right)\right] \theta_{*}^{2}}{2\left(u_{*}+b\right)^{2}\left(\theta_{*}-2\right)^{2}} \\
& +\frac{2\left[c-u_{*}\left(1-u_{*}\right)\right] \theta_{*}+\left(1-b-2 u_{*}\right)\left[\left(1-u_{*}\right)\left(1+b+2 u_{*}\right)-2 c\right] \theta_{*}}{2\left(u_{*}+b\right)^{2}\left(\theta_{*}-2\right)} \\
& -\frac{c^{2}\left(1-u_{*}\right) \theta_{*}^{3}}{6\left(u_{*}+b\right)^{2}\left(\theta_{*}-2\right)^{3}} \\
& a_{210}=\frac{6\left(1-u_{*}\right)\left[b+(1-b-2 c) u_{*}\right]+\left(1-b-2 u_{*}\right)^{2}\left[b+(2+b+2 c) u_{*}\right]}{2\left(u_{*}+b\right)^{3}} \\
& +\frac{c\left[c-(1-u)\left(1+b+2 u_{*}\right)\right] \theta_{*}^{2}}{2\left(u_{*}+b\right)^{2}\left(\theta_{*}-2\right)^{2}}
\end{aligned}
$$




$$
\begin{aligned}
& +\frac{2\left[c-u_{*}\left(1-u_{*}\right)\right] \theta_{*}+\left(1-b-2 u_{*}\right)\left[\left(1-u_{*}\right)\left(1+b+2 u_{*}\right)-2 c\right] \theta_{*}}{\left(u_{*}+b\right)^{2}\left(\theta_{*}-2\right)} \\
& +\frac{c\left[c-(1-u)\left(1+b+2 u_{*}\right)\right] \theta_{*}^{2}}{\left(u_{*}+b\right)^{2}\left(\theta_{*}-2\right)\left(\lambda_{2}-1+\theta_{*}\right)} \\
& +\frac{2\left[c-u_{*}\left(1-u_{*}\right)\right] \theta_{*}+\left(1-b-2 u_{*}\right)\left[\left(1-u_{*}\right)\left(1+b+2 u_{*}\right)-2 c\right] \theta_{*}}{2\left(u_{*}+b\right)^{2}\left(\lambda_{2}-1+\theta_{*}\right)} \\
& -\frac{c^{2}\left(1-u_{*}\right) \theta_{*}^{3}}{2\left(u_{*}+b\right)^{2}\left(\theta_{*}-2\right)^{2}\left(\lambda_{2}-1+\theta_{*}\right)}, \\
& a_{120}=\frac{6\left(1-u_{*}\right)\left[b+(1-b-2 c) u_{*}\right]+\left(1-b-2 u_{*}\right)^{2}\left[b+(2+b+2 c) u_{*}\right]}{2\left(u_{*}+b\right)^{3}} \\
& +\frac{c\left[c-(1-u)\left(1+b+2 u_{*}\right)\right] \theta_{*}^{2}}{\left(u_{*}+b\right)^{2}\left(\theta_{*}-2\right)\left(\lambda_{2}-1+\theta_{*}\right)} \\
& +\frac{2\left[c-u_{*}\left(1-u_{*}\right)\right] \theta_{*}+\left(1-b-2 u_{*}\right)\left[\left(1-u_{*}\right)\left(1+b+2 u_{*}\right)-2 c\right] \theta_{*}}{2\left(u_{*}+b\right)^{2}\left(\theta_{*}-2\right)} \\
& +\frac{c\left[c-(1-u)\left(1+b+2 u_{*}\right)\right] \theta_{*}^{2}}{2\left(u_{*}+b\right)^{2}\left(\lambda_{2}-1+\theta_{*}\right)^{2}} \\
& +\frac{2\left[c-u_{*}\left(1-u_{*}\right)\right] \theta_{*}+\left(1-b-2 u_{*}\right)\left[\left(1-u_{*}\right)\left(1+b+2 u_{*}\right)-2 c\right] \theta_{*}}{\left(u_{*}+b\right)^{2}\left(\lambda_{2}-1+\theta_{*}\right)} \\
& -\frac{c^{2}\left(1-u_{*}\right) \theta_{*}^{3}}{2\left(u_{*}+b\right)^{2}\left(\theta_{*}-2\right)\left(\lambda_{2}-1+\theta_{*}\right)^{2}}, \\
& a_{030}=\frac{6\left(1-u_{*}\right)\left[b+(1-b-2 c) u_{*}\right]+\left(1-b-2 u_{*}\right)^{2}\left[b+(2+b+2 c) u_{*}\right]}{6\left(u_{*}+b\right)^{3}} \\
& +\frac{c\left[c-(1-u)\left(1+b+2 u_{*}\right)\right] \theta_{*}^{2}}{2\left(u_{*}+b\right)^{2}\left(\lambda_{2}-1+\theta_{*}\right)^{2}} \\
& +\frac{2\left[c-u_{*}\left(1-u_{*}\right)\right] \theta_{*}+\left(1-b-2 u_{*}\right)\left[\left(1-u_{*}\right)\left(1+b+2 u_{*}\right)-2 c\right] \theta_{*}}{2\left(u_{*}+b\right)^{2}\left(\lambda_{2}-1+\theta_{*}\right)} \\
& -\frac{c^{2}\left(1-u_{*}\right) \theta_{*}^{3}}{6\left(u_{*}+b\right)^{2}\left(\lambda_{2}-1+\theta_{*}\right)^{3}} \\
& b_{200}=\frac{\theta_{*}\left(\theta_{*}-2\right)}{2 u_{*}}+\frac{\left(2-\theta_{*}\right) \theta_{*}^{2}}{u_{*}\left(\theta_{*}-2\right)}+\frac{\left(\theta_{*}-2\right) \theta_{*}^{3}}{2 u_{*}\left(\theta_{*}-2\right)^{2}} \text {, } \\
& b_{110}=\frac{\theta_{*}\left(\theta_{*}-2\right)}{u_{*}}+\frac{\left(2-\theta_{*}\right) \theta_{*}^{2}}{u_{*}\left(\lambda_{2}-1+\theta_{*}\right)}+\frac{\left(\theta_{*}-2\right) \theta_{*}^{3}}{u_{*}\left(\theta_{*}-2\right)\left(\lambda_{2}-1+\theta_{*}\right)}+\frac{\left(2-\theta_{*}\right) \theta_{*}^{2}}{u_{*}\left(\theta_{*}-2\right)} \text {, } \\
& b_{101}=1-\frac{\theta_{*}}{\theta_{*}-2}, \\
& b_{020}=\frac{\theta_{*}\left(\theta_{*}-2\right)}{2 u_{*}}+\frac{\left(2-\theta_{*}\right) \theta_{*}^{2}}{u_{*}\left(\lambda_{2}-1+\theta_{*}\right)}+\frac{\left(\theta_{*}-2\right) \theta_{*}^{3}}{2 u_{*}\left(\lambda_{2}-1+\theta_{*}\right)^{2}}, \\
& b_{011}=1-\frac{\theta_{*}}{\lambda_{2}-1+\theta_{*}} \text {, } \\
& b_{300}=\frac{6 \theta_{*}-6 \theta_{*}^{2}+\theta_{*}^{3}}{6 u_{*}^{2}}+\frac{\left(-4+5 \theta_{*}-\theta_{*}^{2}\right) \theta_{*}^{2}}{2 u_{*}^{2}\left(\theta_{*}-2\right)}+\frac{\left(2-4 \theta_{*}+\theta_{*}^{2}\right) \theta_{*}^{3}}{2 u_{*}^{2}\left(\theta_{*}-2\right)^{2}}+\frac{\left(3-\theta_{*}\right) \theta_{*}^{5}}{6 u_{*}^{2}\left(\theta_{*}-2\right)^{3}} \text {, } \\
& b_{210}=\frac{6 \theta_{*}-6 \theta_{*}^{2}+\theta_{*}^{3}}{2 u_{*}^{2}}+\frac{\left(-4+5 \theta_{*}-\theta_{*}^{2}\right) \theta_{*}^{2}}{u_{*}^{2}\left(\theta_{*}-2\right)}+\frac{\left(2-4 \theta_{*}+\theta_{*}^{2}\right) \theta_{*}^{3}}{2 u_{*}^{2}\left(\theta_{*}-2\right)^{2}}+\frac{\left(-4+5 \theta_{*}-\theta_{*}^{2}\right) \theta_{*}^{2}}{2 u_{*}^{2}\left(\lambda_{2}-1+\theta_{*}\right)} \\
& +\frac{\left(2-4 \theta_{*}+\theta_{*}^{2}\right) \theta_{*}^{3}}{u_{*}^{2}\left(\theta_{*}-2\right)\left(\lambda_{2}-1+\theta_{*}\right)}+\frac{\left(3-\theta_{*}\right) \theta_{*}^{5}}{2 u_{*}^{2}\left(\theta_{*}-2\right)^{2}\left(\lambda_{2}-1+\theta_{*}\right)},
\end{aligned}
$$




$$
\begin{aligned}
b_{201}= & \frac{\theta_{*}-1}{u_{*}}+\frac{\left(2-2 \theta_{*}\right) \theta_{*}}{u_{*}\left(\theta_{*}-2\right)}+\frac{\left(\theta_{*}-1\right) \theta_{*}^{2}}{u_{*}\left(\theta_{*}-2\right)^{2}}, \\
b_{120}= & \frac{6 \theta_{*}-6 \theta_{*}^{2}+\theta_{*}^{3}}{2 u_{*}^{2}}+\frac{\left(-4+5 \theta_{*}-\theta_{*}^{2}\right) \theta_{*}^{2}}{2 u_{*}^{2}\left(\theta_{*}-2\right)}+\frac{\left(-4+5 \theta_{*}-\theta_{*}^{2}\right) \theta_{*}^{2}}{u_{*}^{2}\left(\lambda_{2}-1+\theta_{*}\right)}+\frac{\left(2-4 \theta_{*}+\theta_{*}^{2}\right) \theta_{*}^{3}}{2 u_{*}^{2}\left(\lambda_{2}-1+\theta_{*}\right)^{2}} \\
& +\frac{\left(2-4 \theta_{*}+\theta_{*}^{2}\right) \theta_{*}^{3}}{u_{*}^{2}\left(\theta_{*}-2\right)\left(\lambda_{2}-1+\theta_{*}\right)}+\frac{\left(3-\theta_{*}\right) \theta_{*}^{5}}{2 u_{*}^{2}\left(\theta_{*}-2\right)\left(\lambda_{2}-1+\theta_{*}\right)^{2}}, \\
b_{111}= & \frac{2\left(\theta_{*}-1\right)}{u_{*}}+\frac{\left(2-2 \theta_{*}\right) \theta_{*}}{u_{*}\left(\theta_{*}-2\right)}+\frac{\left(2-2 \theta_{*}\right) \theta_{*}}{u_{*}\left(\lambda_{2}-1+\theta_{*}\right)}+\frac{2\left(\theta_{*}-1\right) \theta_{*}^{2}}{u_{*}\left(\theta_{*}-2\right)\left(\lambda_{2}-1+\theta_{*}\right)}, \\
b_{030}= & \frac{6 \theta_{*}-6 \theta_{*}^{2}+\theta_{*}^{3}}{6 u_{*}^{2}}+\frac{\left(-4+5 \theta_{*}-\theta_{*}^{2}\right) \theta_{*}^{2}}{2 u_{*}^{2}\left(\lambda_{2}-1+\theta_{*}\right)}+\frac{\left(2-4 \theta_{*}+\theta_{*}^{2}\right) \theta_{*}^{3}}{2 u_{*}^{2}\left(\lambda_{2}-1+\theta_{*}\right)^{2}}+\frac{\left(3-\theta_{*}\right) \theta_{*}^{5}}{6 u_{*}^{2}\left(\lambda_{2}-1+\theta_{*}\right)^{3}}, \\
b_{021}= & \frac{\theta_{*}-1}{u_{*}}+\frac{\left(2-2 \theta_{*}\right) \theta_{*}}{u_{*}\left(\lambda_{2}-1+\theta_{*}\right)}+\frac{\left(\theta_{*}-1\right) \theta_{*}^{2}}{u_{*}\left(\lambda_{2}-1+\theta_{*}\right)^{2}} .
\end{aligned}
$$

From the center manifold theory of discrete system we know that there exists a local manifold of model (21) [12]. The local manifold has the following expansion:

$$
\begin{aligned}
v_{1}(t)= & h\left(u_{1}(t), \theta_{1}(t)\right) \\
= & m_{1} u_{1}^{2}(t)+m_{2} u_{1}(t) \theta_{1}(t)+m_{3} \theta_{1}^{2}(t)+m_{4} u_{1}^{3}(t)+m_{5} u_{1}^{2}(t) \theta_{1}(t) \\
& +m_{6} u_{1}(t) \theta_{1}^{2}(t)+m_{7} \theta_{1}^{3}(t)+o\left(\left(\left|u_{1}\right|+\left|\theta_{1}\right|\right)^{3}\right) .
\end{aligned}
$$

After substituting the expansion into model (21) and using the invariant property of the local manifold, the straightforward and careful calculation gives $m_{2}=m_{3}=m_{6}=m_{7}=0$, and

$$
\begin{aligned}
& m_{1}=\frac{\lambda_{2}-1+\theta_{*}}{1+\lambda_{2}}\left(a_{200}-\frac{\theta_{*}-2}{\theta_{*}} b_{200}\right), \\
& m_{4}=-\frac{2 m_{1}\left(\theta_{*}-2\right)}{1+\lambda_{2}}\left(-a_{200}+\frac{\lambda_{2}-1+\theta_{*}}{\theta_{*}} b_{200}\right)-\frac{\lambda_{2}-1+\theta_{*}}{1+\lambda_{2}}\left(a_{300}-\frac{\theta_{*}-2}{\theta_{*}} b_{300}\right), \\
& m_{5}=\frac{2 m_{1}\left(\theta_{*}-2\right)\left(\lambda_{2}-1+\theta_{*}\right) b_{101}}{\theta_{*}\left(1+\lambda_{2}\right)}-\frac{\left(\lambda_{2}-1+\theta_{*}\right)\left(\theta_{*}-2\right)}{\theta_{*}\left(1+\lambda_{2}\right)} b_{201} .
\end{aligned}
$$

From the second equation of model (21) we know that $\theta(t)$ is always constant. Therefore, the one dimensional model induced by the center manifold is

$$
u_{1}(t+1)=G\left(u_{1}(t), \theta_{1}\right)
$$

where

$$
\begin{aligned}
G= & -u_{1}(t)+\frac{\left(\lambda_{2}-1+\theta_{*}\right)\left(\theta_{*}-2\right) b_{101}}{\theta_{*}\left(1+\lambda_{2}\right)} \theta_{1} u_{1}+\frac{\theta_{*}-2}{1+\lambda_{2}}\left(-a_{200}+\frac{\lambda_{2}-1+\theta_{*}}{\theta_{*}} b_{200}\right) u_{1}^{2}(t) \\
& +\frac{\left(\lambda_{2}-1+\theta_{*}\right)\left(\theta_{*}-2\right) b_{201}}{\theta_{*}\left(1+\lambda_{2}\right)} \theta_{1} u_{1}^{2}+\frac{\left(\lambda_{2}-1+\theta_{*}\right)\left(\theta_{*}-2\right) b_{011}}{\theta_{*}\left(1+\lambda_{2}\right)} m_{1} \theta_{1} u_{1}^{2}(t) \\
& +\frac{\left(\lambda_{2}-1+\theta_{*}\right)\left(\theta_{*}-2\right) b_{011}}{\theta_{*}\left(1+\lambda_{2}\right)} m_{5} \theta_{1}^{2} u_{1}^{2}(t)+\frac{\theta_{*}-2}{1+\lambda_{2}}\left(-a_{300}+\frac{\lambda_{2}-1+\theta_{*}}{\theta_{*}} b_{300}\right) u_{1}^{3}(t) \\
& +\frac{\theta_{*}-2}{1+\lambda_{2}}\left(-a_{110}+\frac{\lambda_{2}-1+\theta_{*}}{\theta_{*}} b_{110}\right) m_{1} u_{1}^{3}(t)+\frac{\left(\lambda_{2}-1+\theta_{*}\right)\left(\theta_{*}-2\right) b_{111}}{\theta_{*}\left(1+\lambda_{2}\right)} m_{1} \theta_{1} u_{1}^{3}
\end{aligned}
$$




$$
\begin{aligned}
& +\frac{\left(\lambda_{2}-1+\theta_{*}\right)\left(\theta_{*}-2\right) b_{011}}{\theta_{*}\left(1+\lambda_{2}\right)} m_{4} \theta_{1} u_{1}^{3} \\
& +\frac{\theta_{*}-2}{1+\lambda_{2}}\left(-a_{110}+\frac{\lambda_{2}-1+\theta_{*}}{\theta_{*}} b_{110}\right) m_{5} \theta_{1} u_{1}^{3} .
\end{aligned}
$$

It is not difficult to verify that $G\left(0, \theta_{1}\right)=0, \frac{\partial G(0,0)}{\partial u_{1}}=-1$, and

$$
\begin{aligned}
& \frac{\partial^{2} G(0,0)}{\partial u_{1} \partial \theta_{1}}=\frac{\left(\lambda_{2}-1+\theta_{*}\right)\left(\theta_{*}-2\right)}{\theta_{*}\left(1+\lambda_{2}\right)} b_{101}=-\frac{u_{*}(1+b+c)^{2}}{u_{*}(1+b+(b+c)(1+b+2 c))-2 b c}<0, \\
& \beta=\frac{\partial^{3} G^{2}(0,0)}{\partial u^{3}}=-\frac{12\left(\theta_{*}-2\right)}{1+\lambda_{2}}\left(-a_{200}+\frac{\lambda_{2}-1+\theta_{*}}{\theta_{*}} b_{200}\right) \neq 0 .
\end{aligned}
$$

Therefore, model (3) will undergo a flip bifurcation at $E_{2}\left(u_{*}, u_{*}\right)$, and the bifurcation solution of period two is stable (unstable) when $\beta<0(\beta>0)$ [13].

We use numerical simulation to demonstrate the flip bifurcation of model (3). When parameter values $b=1$ and $c=2$ are taken, then the positive equilibrium of (3) is $E(\sqrt{2}$ $1, \sqrt{2}-1)$, the critical value of $\theta_{*}=3$. The positive equilibrium $E(\sqrt{2}-1, \sqrt{2}-1)$ is stable when $0<\theta<3$. $E(\sqrt{2}-1, \sqrt{2}-1)$ is unstable if $\theta>3$. The calculation shows that

$$
\begin{aligned}
G\left(u_{1}, \theta_{1}\right)= & -u_{1}-1.547 \theta_{1} u_{1}+13.591 u_{1}^{2}(t)+14.938 \theta_{1} u_{1}^{2}+8.422 \theta_{1} u_{1}^{2}(t) \\
& -17.885 \theta_{1}^{2} u_{1}^{2}(t)-27.557 u_{1}^{3}(t)-217.889 u_{1}^{3}(t)-162.651 \theta_{1} u_{1}^{3} \\
& -213.622 \theta_{1} u_{1}^{3}+462.730 \theta_{1} u_{1}^{3}+o\left(\rho^{3}\right) .
\end{aligned}
$$

Further calculation shows that

$$
\begin{aligned}
& G\left(0, \theta_{1}\right)=0, \quad \frac{\partial G(0,0)}{\partial u_{1}}=-1, \quad \frac{\partial^{2} G(0,0)}{\partial u_{1} \partial \theta_{1}}=-1.547<0, \\
& \beta=\frac{\partial^{3} G^{2}(0,0)}{\partial u^{3}}=-12 \times 13.591<0 .
\end{aligned}
$$

From Theorem 3.1 we know that there exists a flip bifurcation of model (3) when $\theta_{*}<\theta<$ $\theta_{*}+\varepsilon$, and the period two cycle is stable. The numerical simulation shows that the period two cycle of model (3) may be globally asymptotically stable when $\theta>\theta_{*}$ and $\theta-\theta_{*}$ is small.

Figure 3 shows the flip bifurcation of model (3) and its stability. For the subplots in the left column, the parameters are $b=1, c=2$, and $\theta=3.01 . E_{1}(0.3760,0.3102)$ and $E_{2}(0.4471,0.5254)$ are two points at the period two cycle of model (3) for those parameters. The solution of model (3) with initial conditions $u(0)=0.447, v(1)=0.525$ tends to the period two cycle. For the subplots in the right column, the parameters are $b=1$, $c=2$, and $\theta=3.1 . E_{1}(0.3115,0.1659)$ and $E_{2}(0.4481,0.7067)$ are the period two cycle of model (3) for those parameters. The solution of model (3) with the same initial conditions $u(0)=0.447, v(1)=0.525$ tends to the period two cycle quickly. The simulations show that the magnitude of the period two cycle of model (3) increases with the parameter $\theta$, and the period two cycle may be globally asymptotically stable when $\theta>\theta_{*}$ and $\theta-\theta_{*}$ are small. 

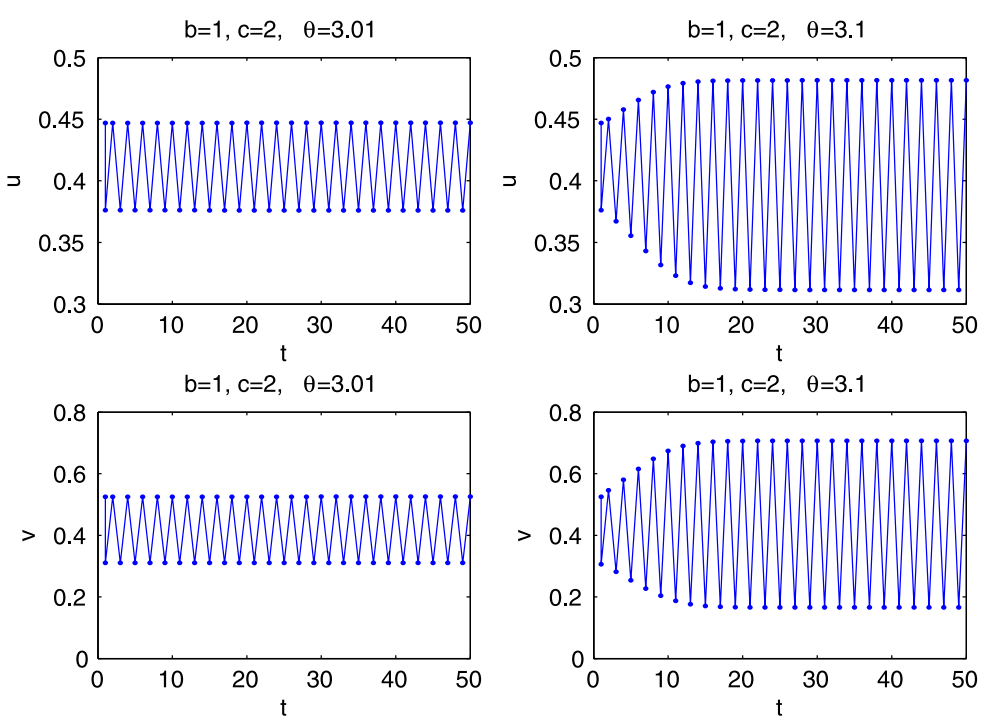

Figure 3 The flip bifurcation of model (3) from the equilibrium point $E\left(u_{*}, u_{*}\right)$.

\subsection{Neimark-Sacker bifurcation}

The Neimark-Sacker bifurcation for the discrete models is similar to the Hopf bifurcation of continuous models. In this subsection we discuss the existence of the Neimark-Sacker bifurcation of model (3).

Theorem 3.2 If $\alpha \neq 0$, then model (3) will undergo a Neimark-Sacker bifurcation at $E\left(u_{*}, u_{*}\right)$ when $\theta=\frac{1-b-2 u_{*}}{1-u_{*}}$ with $1-b-2 u_{*}>0$, where $\alpha$ is defined in the proof.

Proof Let $u_{1}(t)=u(t)-u_{*}$ and $v_{1}(t)=v(t)-u_{*}$, then the equilibrium $E\left(u_{*}, u_{*}\right)$ is transformed into the origin, we have

$$
\left\{\begin{array}{l}
u_{1}(t+1)=\left(u_{1}(t)+u_{*}\right) \exp \left(1-\left(u_{1}(t)+u_{*}\right)-\frac{c\left(v_{1}(t)+u_{*}\right)}{u_{1}(t)+u_{*}+b}\right)-u_{*}, \\
v_{1}(t+1)=\left(v_{1}(t)+u_{*}\right) \exp \left(\theta\left(1-\frac{v_{1}(t)+u_{*}}{u_{1}(t)+u_{*}}\right)\right)-u_{*} .
\end{array}\right.
$$

The Taylor expression of model $(25)$ at $\left(u_{1}(t), v_{1}(t)\right)=(0,0)$ to the third order is

$$
\left\{\begin{array}{l}
u_{1}(t+1)=\frac{(b+2 c) u_{*}-b}{u_{*}+b} u_{1}(t)-\left(1-u_{*}\right) v_{1}(t)+P_{1}\left(u_{1}, v_{1}\right), \\
v_{1}(t+1)=\theta u_{1}(t)+(1-\theta) v_{1}(t)+P_{2}\left(u_{1}, v_{1}\right),
\end{array}\right.
$$

where

$$
\begin{aligned}
P_{1}\left(u_{1}, v_{1}\right)= & \frac{\left(2 c-1-(b+2 c)\left(b+2 u_{*}\right)\right) u_{*}}{2\left(u_{*}+b\right)^{2}} u_{1}^{2}+\frac{(b+2 c) u_{*}-(b+c)}{u_{*}+b} u_{1} v_{1}+\frac{c\left(1-u_{*}\right)}{2\left(u_{*}+b\right)} v_{1}^{2} \\
& +\frac{6\left(1-u_{*}\right)\left[b+(1-b-2 c) u_{*}\right]+\left(1-b-2 u_{*}\right)^{2}\left[b+(2+b+2 c) u_{*}\right]}{6\left(u_{*}+b\right)^{3}} u_{1}^{3} \\
& +\frac{2\left[c-u_{*}\left(1-u_{*}\right)\right]+\left(1-b-2 u_{*}\right)\left[\left(1-u_{*}\right)\left(1+b+2 u_{*}\right)-2 c\right]}{2\left(u_{*}+b\right)^{2}} u_{1}^{2} v_{1} \\
& +\frac{c\left[c-(1-u)\left(1+b+2 u_{*}\right)\right]}{2\left(u_{*}+b\right)^{2}} u_{1} v_{1}^{2}-\frac{c^{2}\left(1-u_{*}\right)}{6\left(u_{*}+b\right)^{2}} v_{1}^{3}+o\left(\left(\left|u_{1}\right|+\left|v_{1}\right|\right)^{3}\right)
\end{aligned}
$$




$$
\begin{aligned}
P_{2}\left(u_{1}, v_{1}\right)= & \frac{\theta(\theta-2)}{2 u_{*}} u_{1}^{2}+\frac{\theta(2-\theta)}{u_{*}} u_{1} v_{1}+\frac{\theta(\theta-2)}{2 u_{*}} v_{1}^{2} \\
& +\frac{\theta\left(\theta^{2}-6 \theta+6\right)}{6 u_{*}^{2}} u_{1}^{3}-\frac{\theta\left(\theta^{2}-5 \theta+4\right)}{2 u_{*}^{2}} u_{1}^{2} v_{1} \\
& +\frac{\theta\left(\theta^{2}-4 \theta+2\right)}{2 u_{*}^{2}} u_{1} v_{1}^{2}-\frac{\theta^{2}(\theta-3)}{6 u_{*}} v_{1}^{3}+o\left(\left(\left|u_{1}\right|+\left|v_{1}\right|\right)^{3}\right) .
\end{aligned}
$$

When $\theta_{01}<\theta<\theta_{02}$, the eigenvalues of the linearized matrix of model (26) are $\lambda_{1}=\bar{\lambda}_{2}=$ $\mu+i \omega$, and $\left|\lambda_{1,2}\right|=\sqrt{\frac{(1-\theta)\left((b+2 c) u_{*}-b\right)}{u_{*}+b}+\left(1-u_{*}\right) \theta}$, where

$$
\begin{aligned}
& \theta_{01}=3-2 u_{*}-\frac{(b+2 c) u_{*}-b}{u_{*}+b}-2 \sqrt{\left(1-u_{*}\right)\left(2-u_{*}-\frac{(b+2 c) u_{*}-b}{u_{*}+b}\right)}, \\
& \theta_{02}=3-2 u_{*}-\frac{(b+2 c) u_{*}-b}{u_{*}+b}+2 \sqrt{\left(1-u_{*}\right)\left(2-u_{*}-\frac{(b+2 c) u_{*}-b}{u_{*}+b}\right)}, \\
& \mu=\frac{1}{2}\left(\frac{(b+2 c) u_{*}-b}{u_{*}+b}+1-\theta\right), \\
& \omega=\frac{1}{2} \sqrt{\frac{4(1-\theta)\left((b+2 c) u_{*}-b\right)}{u_{*}+b}+4 \theta\left(1-u_{*}\right)-\left(\frac{(b+2 c) u_{*}-b}{u_{*}+b}+1-\theta\right)^{2}} .
\end{aligned}
$$

Let $\theta$ be the bifurcation parameter and $\theta_{0}=\frac{1-b-2 u_{*}}{1-u_{*}}$. The expression of $u_{*}$ and the straightforward calculation yield that

$$
\left|\lambda_{1,2}\right|_{\theta=\theta_{0}}=1,\left.\quad \frac{d\left|\lambda_{1,2}\right|}{d \theta}\right|_{\theta=\theta_{0}}=\frac{1}{2}\left(1-u_{*}-\frac{(b+2 c) u_{*}-b}{u_{*}+b}\right)=\frac{b-(b+c) u_{*}}{2\left(u_{*}+b\right)}<0 .
$$

Further calculation shows that $\lambda_{1,2}^{m} \neq 1$ for $\theta=\theta_{0}$ and $m=1,2,3,4$. Let $T=\left(\begin{array}{cc}t_{11} & t_{12} \\ t_{21} & t_{22}\end{array}\right)$ with $t_{11}=1, t_{12}=0, t_{21}=\frac{\mu-(1-\theta)}{1-u_{*}}$, and $t_{22}=\frac{\omega}{1-u_{*}}$. By performing the transformation

$$
\left(\begin{array}{l}
u_{1}(t) \\
v_{1}(t)
\end{array}\right)=T\left(\begin{array}{l}
u_{2}(t) \\
v_{2}(t)
\end{array}\right)
$$

we obtain

$$
\left(\begin{array}{l}
u_{2}(t+1) \\
v_{2}(t+1)
\end{array}\right)=\left(\begin{array}{cc}
\mu & -\omega \\
\omega & \mu
\end{array}\right)\left(\begin{array}{l}
u_{2}(t) \\
v_{2}(t)
\end{array}\right)+\left(\begin{array}{l}
Q_{1}\left(u_{2}, v_{2}\right) \\
Q_{2}\left(u_{2}, v_{2}\right)
\end{array}\right)
$$

where

$$
\begin{aligned}
& Q_{1}\left(u_{2}, v_{2}\right) \\
& =\left(\frac{\left(2 c-1-(b+2 c)\left(b+2 u_{*}\right)\right) u_{*}}{2\left(u_{*}+b\right)^{2}}+\frac{(b+2 c) u_{*}-(b+c)}{u_{*}+b} t_{21}+\frac{c\left(1-u_{*}\right)}{2\left(u_{*}+b\right)} t_{21}^{2}\right) u_{2}^{2} \\
& \quad+\left(\frac{(b+2 c) u_{*}-(b+c)}{u_{*}+b} t_{22}+\frac{c\left(1-u_{*}\right)}{\left(u_{*}+b\right)} t_{21} t_{22}\right) u_{2} v_{2}+\frac{c\left(1-u_{*}\right)}{2\left(u_{*}+b\right)} t_{22}^{2} v_{2}^{2} \\
& \quad+\left(\frac{6\left(1-u_{*}\right)\left[b+(1-b-2 c) u_{*}\right]+\left(1-b-2 u_{*}\right)^{2}\left[b+(2+b+2 c) u_{*}\right]}{6\left(u_{*}+b\right)^{3}}\right.
\end{aligned}
$$




$$
\begin{aligned}
&+\frac{2\left[c-u_{*}\left(1-u_{*}\right)\right]+\left(1-b-2 u_{*}\right)\left[\left(1-u_{*}\right)\left(1+b+2 u_{*}\right)-2 c\right]}{2\left(u_{*}+b\right)^{2}} t_{21} \\
&\left.+\frac{c\left[c-(1-u)\left(1+b+2 u_{*}\right)\right]}{2\left(u_{*}+b\right)^{2}} t_{21}^{2}-\frac{c^{2}\left(1-u_{*}\right)}{6\left(u_{*}+b\right)^{2}} t_{21}^{3}\right) u_{2}^{3} \\
&+\left(\frac{c\left[c-(1-u)\left(1+b+2 u_{*}\right)\right]}{\left(u_{*}+b\right)^{2}} t_{21} t_{22}-\frac{c^{2}\left(1-u_{*}\right)}{2\left(u_{*}+b\right)^{2}} t_{21}^{2} t_{22}\right) u_{2}^{2} v_{2} \\
&+\left(\frac{c\left[c-(1-u)\left(1+b+2 u_{*}\right)\right]}{2\left(u_{*}+b\right)^{2}} t_{22}^{2}-\frac{c^{2}\left(1-u_{*}\right)}{2\left(u_{*}+b\right)^{2}} t_{21} t_{22}^{2}\right) u_{2} v_{2}^{2}-\frac{c^{2}\left(1-u_{*}\right)}{6\left(u_{*}+b\right)^{2}} t_{22}^{3} v_{2}^{3}, \\
& P\left(u_{2}, v_{2}\right)\left(\frac{\theta(\theta-2)}{2 u_{*}}+\frac{\theta(2-\theta)}{u_{*}} t_{21}+\frac{\theta(\theta-2)}{2 u_{*}} t_{21}^{2}\right) u_{2}^{2}+\left(\frac{\theta(2-\theta)}{u_{*}} t_{22}+\frac{\theta(\theta-2)}{u_{*}} t_{21} t_{22}\right) u_{2} v_{2} \\
&+\frac{\theta(\theta-2)}{2 u_{*}} t_{22}^{2} v_{2}^{2}+\left(\frac{\theta\left(\theta^{2}-6 \theta+6\right)}{6 u_{*}^{2}}-\frac{\theta\left(\theta^{2}-5 \theta+4\right)}{2 u_{*}^{2}} t_{21}\right. \\
&\left.+\frac{\theta\left(\theta^{2}-4 \theta+2\right)}{2 u_{*}^{2}} t_{21}^{2}-\frac{\theta^{2}(\theta-3)}{6 u_{*}} t_{21}^{3}\right) u_{2}^{3} \\
&+\left(-\frac{\theta\left(\theta^{2}-5 \theta+4\right)}{2 u_{*}^{2}} t_{22}+\frac{\theta\left(\theta^{2}-4 \theta+2\right)}{u_{*}^{2}} t_{21} t_{22}-\frac{\theta^{2}(\theta-3)}{2 u_{*}} t_{21}^{2} t_{22}\right) u_{2}^{2} v_{2} \\
&+\left(\frac{\theta\left(\theta^{2}-4 \theta+2\right)}{2 u_{*}^{2}} t_{22}^{2}-\frac{\theta^{2}(\theta-3)}{2 u_{*}} t_{21} t_{22}^{2}\right) u_{2} v_{2}^{2}-\frac{\theta^{2}(\theta-3)}{6 u_{*}} t_{22}^{3} v_{2}^{3} \\
& Q_{2}\left(u_{2}, v_{2}\right)=-\frac{t_{21}}{t_{22}} Q_{1}+\frac{P\left(u_{2}, v_{2}\right)}{t_{22}} .
\end{aligned}
$$

From Theorem 3.5.3 of [14] we know that the existence of a Neimark-Sacker bifurcation can be determined by the quantity $\alpha$, where

$$
\alpha=-\operatorname{Re}\left[\frac{(1-2 \lambda) \bar{\lambda}^{2}}{1-\lambda} l_{11} l_{20}\right]-\frac{1}{2}\left|l_{11}\right|^{2}-\left|l_{02}\right|^{2}+\operatorname{Re}\left(\bar{\lambda} l_{21}\right),
$$

and

$$
\begin{aligned}
l_{20}= & \frac{1}{8}\left[\left(Q_{1 u_{2} u_{2}}-Q_{1 v_{2} v_{2}}+2 Q_{2 u_{2} v_{2}}\right)+i\left(Q_{2 u_{2} u_{2}}-Q_{2 v_{2} v_{2}}-2 Q_{1 u_{2} v_{2}}\right)\right], \\
l_{11}= & \frac{1}{4}\left[Q_{1 u_{2} u_{2}}+Q_{1 v_{2} v_{2}}+i\left(Q_{2 u_{2} u_{2}}+Q_{2 v_{2} v_{2}}\right)\right], \\
l_{02}= & \frac{1}{8}\left[\left(Q_{1 u_{2} u_{2}}-Q_{1 v_{2} v_{2}}-2 Q_{2 u_{2} v_{2}}\right)+i\left(Q_{2 u_{2} u_{2}}-Q_{2 v_{2} v_{2}}+2 Q_{1 u_{2} v_{2}}\right)\right], \\
l_{21}= & \frac{1}{16}\left[\left(Q_{1 u_{2} u_{2} u_{2}}+Q_{1 u_{2} v_{2} v_{2}}+Q_{2 u_{2} u_{2} v_{2}}+Q_{2 v_{2} v_{2} v_{2}}\right)\right. \\
& \left.+i\left(Q_{2 u_{2} u_{2} u_{2}}+Q_{2 u_{2} v_{2} v_{2}}-Q_{1 u_{2} u_{2} v_{2}}-Q_{1 v_{2} v_{2} v_{2}}\right)\right] .
\end{aligned}
$$

Using the Neimark-Sacker bifurcation theorem in [14], we obtain that there exists a Neimark-Sacker bifurcation when $\alpha \neq 0$ and $\theta$ passes through $\theta_{0}$.

We use numerical simulation to demonstrate the Neimark-Sacker bifurcation of model (3). When parameter values are taken to be $b=0.1001$ and $c=0.8$, then the positive equi- 


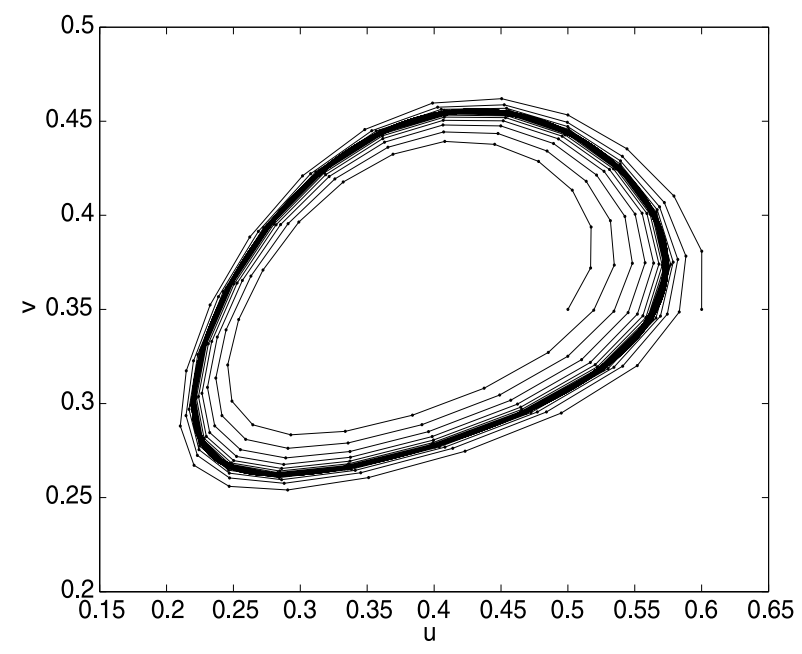

Figure 4 The Neimark-Sacker bifurcation of model (3) at the equilibrium point $E_{2}$.

librium of (3) is $E(0.3703,0.3703)$, the critical value $\theta_{0}=0.2531$. When $\theta=\theta_{0}$, model (3) will undergo a Neimark-Sacker bifurcation at $E\left(u_{*}, u_{*}\right)$ (see Figure 4 ).

\section{Conclusion and discussion}

The predator-prey model with Holling-Tanner functional response can give better prediction for some interacting species. The model also exhibits more complicated dynamics. We have studied the dynamical behaviors of a discrete prey-predator model with HollingTanner functional response. We have obtained sufficient conditions for the stability of the positive equilibrium, the existence of a flip bifurcation and a Neimark-Sacker bifurcation. The numerical simulations show that the model possesses more complicated dynamics. For example, if we take $c=2, b=1$, then the positive equilibrium is $E(\sqrt{2}-1, \sqrt{2}-1)$. The stability condition of $E(\sqrt{2}-1, \sqrt{2}-1)$ is $0<\theta<3$. The numerical simulation shows that model (3) undergoes a process from periodic doubling to chaos (see Figure 5).

The horizontal axis in Figure 5 is the parameter $\theta$, and the vertical axis is the limiting points of $u(t)$. When $0<\theta<3$, there is only one limiting point of $u(t)$, which is the value of the positive equilibrium. When $3<\theta<3.387$, the positive equilibrium loses its stability and a stable period two cycle appears. When $3.387<\theta<3.496$, the period two cycle loses its stability and a stable period four cycle appears. The period doubling process continues to chaos as $\theta$ increases. The top-left subplot shows a complete bifurcation. Three different domains, $[3.3,3.6] \times[0.4,0.55],[3.3,3.6] \times[0.15,0.4]$, and $[3.85,4.0] \times[0.0,0.7]$, in the bifurcation figure are enlarged and displayed in the other three subplots. Especially, from the bottom-left subplot we can see that there is a stable period three cycle of model (3).

The dynamics of the discrete predator-prey model with Holling-Tanner functional response is much more complicated. We have investigated the local stability of the positive equilibrium and the bifurcation of the model analytically or numerically. There are still many challenging problems on the dynamics of the model. Does the local stability of the positive equilibrium imply its global stability? Are there two invariant closed curves in the neighborhood of the positive equilibrium? The numerical simulations demonstrate that the positive equilibrium may be globally stable if it is locally stable. The numerical simu- 

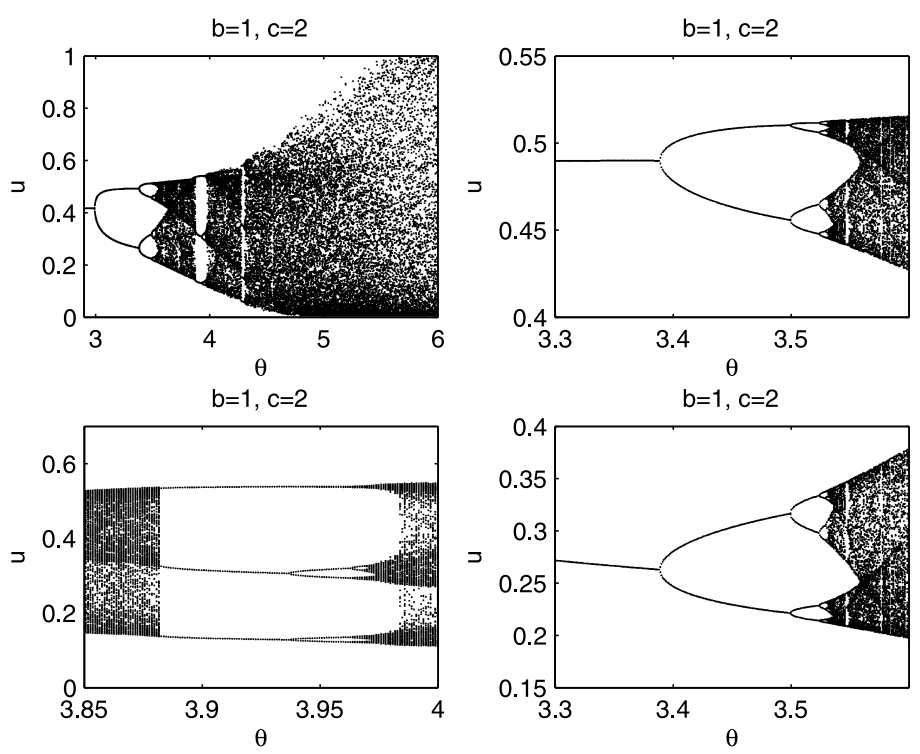

Figure 5 Model (3) may exhibit a process from period doubling to chaos.

lations do not give any information on the existence of two invariant closed curves. We expect that some analytical results can be obtained on those problems in the future.

\section{Competing interests}

We all authors declare that we have no competing interests.

\section{Authors' contributions}

$Y Z$ is responsible for the model formulation and study planning. $\mathrm{HC}$ and $\mathrm{ZY}$ have done the calculation, the proof, the simulation and the application. All authors have read and approved the final manuscript.

\section{Author details}

'Department of Mathematics, Shaanxi University of Science \& Technology, Xi'an, 710021, China. ${ }^{2}$ Department of Applied Mathematics, Xi'an Jiaotong University, Xi'an, 710049, China.

\section{Acknowledgements}

This study was supported by NSFC grant 11301314, by Shaanxi Provincial Education Department grant 2013JK0599, and by Doctoral Research Foundation of Shaanxi University of Science \& Technology grant BJ12-20.

Received: 12 June 2013 Accepted: 16 October 2013 Published: 19 Nov 2013

\section{References}

1. Liz, E, Tkachenko, V, Trofimchuk, S: Global stability in discrete population models with delayed-density dependence. Math. Biosci. 199, 26-37 (2006)

2. Agiza, HN, Elabbasy, EM, El-Metwally, H, Elsadany, AA: Chaotic dynamics of a discrete prey-predator model with Holling type II. Nonlinear Anal., Real World Appl. 10, 116-129 (2009)

3. Elsadany, AA, El-Metwally, HA, Elabbasy, EM, Agsia, HN: Chaos and bifurcation of a nonlinear discrete prey-predator system. Comput. Ecol. Softw. 2(3), 169-180 (2012)

4. Hu, Z, Teng, Z, Zhang, L: Stability and bifurcation analysis of a discrete predator-prey model with nonmonotonic functional response. Nonlinear Anal., Real World Appl. 12, 2356-2377 (2011)

5. Liu, X, Xiao, D: Complex dynamic behaviors of a discrete-time predator-prey system. Chaos Solitons Fractals 32, 80-94 (2007)

6. Celik, C, Duman, O: Allee effect in a discrete-time predator-prey system. Chaos Solitons Fractals 40, 1956-1962 (2009)

7. May, RM: Stability and Complexity in Model Ecosystems. Princeton University Press, Princeton (1973)

8. Sáez, E, González-Olivares, E: Dynamics of a predator-prey model. SIAM J. Appl. Math. 59, 1867-1878 (1999)

9. Tanner, JT: The stability and the intrinsic growth rates of prey and predator populations. Ecology 56, 855-867 (1975)

10. Wollkind, DJ, Collings, JB, Logan, JA: Metastability in a temperature-dependent model system for predator-prey mite outbreak interactions on fruit flies. Bull. Math. Biol. 50, 379-409 (1988)

11. Braza, PA: The bifurcation structure of the Holling-Tanner model for predator-prey interactions using two-timing. SIAM J. Appl. Math. 63(3), 889-904 (2003)

12. Beyn, WJ, Lorenz, J: Center manifolds of dynamical systems under discretization. Numer. Funct. Anal. Optim. 9 381-414 (1987) 
13. Grandmonet, JM: Nonlinear difference equations, bifurcations and chaos: an introduction. Res. Econ. 62, 120-177 (2008)

14. Guckenheimer, J, Holmes, P: Nonlinear Oscillations, Dynamical Systems, and Bifurcations of Vector Fields. Springer New York (1983)

10.1186/1687-1847-2013-330

Cite this article as: Cao et al.: The stability and bifurcation analysis of a discrete Holling-Tanner model. Advances in Difference Equations 2013, 2013:330

Submit your manuscript to a SpringerOpen ${ }^{\circ}$ journal and benefit from:

- Convenient online submission

Rigorous peer review

- Immediate publication on acceptance

Open access: articles freely available online

- High visibility within the field

- Retaining the copyright to your article

Submit your next manuscript at $>$ springeropen.com 